\title{
Statistical Class-Based MFCC Enhancement of Filtered and Band-Limited Speech for Robust ASR
}

\author{
Nicolás Morales $^{(1,2)}$, Doroteo T. Toledano ${ }^{(I)}$, John H. L. Hansen ${ }^{(2)}$, José Colás ${ }^{(I)}$ and Javier Garrido $^{(I)}$ \\ ${ }^{(1)}$ HCTLab-Escuela Politécnica Superior. Univ Autónoma Madrid, SPAIN. \\ ${ }^{(2)}$ Robust Speech Processing Group; Center for Spoken Language Research, Univ. Colorado, \\ Boulder, USA. \\ \{nicolas.morales, doroteo.torre, jose.colas, javier.garrido\}@uam.es, \\ john.hansen@colorado.edu
}

\begin{abstract}
In this paper we address the problem of bandwidth extension from the point of view of ASR. We show that an HMM-based recognition engine trained with full-bandwidth data can successfully perform ASR on limited-bandwidth test data by means of a simple correction scheme over the input feature vectors. In particular we show that results obtained using fullbandwidth HMMs and corrected feature vectors can be comparable to, or even outperform results obtained using limited-bandwidth-trained HMMs. Both results are inferior to those obtained with full-bandwidth HMMs and test data. These results suggest that the effect of channel mismatch on recognition accuracy can be partially compensated with a feature correction scheme, while the loss of information inherent to a limited-bandwidth cannot be compensated.
\end{abstract}

\section{Introduction}

It is well known that mismatched train and test data implies a serious loss in accuracy for Automatic Speech Recognition (ASR). The degradation is so important that some kind of correction needs to be applied to either the model set or the input feature vectors. We focus on the particular case of a base Hidden Markov Model (HMM) set trained with fullbandwidth data performing ASR on limited-bandwidth data. In this paper, we show that such mismatched situation can be efficiently solved by means of a simple polynomial correction applied to the input feature vector.

The problem of bandwidth extension for telephone signal has been extensively analyzed with the objective of rebuilding more natural signals, motivated in many cases by the interest of the telephony industry [1]. Since the typical telephone bandwidth is $300-3400 \mathrm{~Hz}$, it would be desirable to rebuild the whole-band signal (at least $0-8000 \mathrm{~Hz}$. which is normally accepted as full bandwidth for voice) so that the signal sounds more natural. The underlying idea in the recovery process is that the human speech presents a high correlation between the energy distributions in different frequency bands [2]. Under this assumption, the fullbandwidth information is to a certain extent redundant, and a restricted-bandwidth signal could be expanded to a fullbandwidth one. Most efforts in the direction of re-building a full-bandwidth signal use an LPC-based analysis of the signal, create an extended envelope from the restricted-bandwidth version and produce an excitation signal [3] for that envelope. However, our interest being in the speech recognition field, we focus on the MFCC parameters which are widely used for robust ASR. Adopting the same assumption of redundant information, we generate an extended-bandwidth MFCC feature vector from a restricted-bandwidth input MFCC vector.

Another approach to the same problem is to train a new HMM set for each possible frequency bandwidth; or even a ROVER solution can be implemented when several HMM sets are available but none matches perfectly the input characteristics [4]. Both approaches have been used successfully in practical situations; however, they require training and storing of new models. By modifying the input feature vector we expect to obtain similar recognition rates with a simpler and less expensive solution. Feature compensation requires less memory space since a single HMM set is stored and it is easy to train and integrate in an ASR engine thanks to its modularity. It is our interest to be able to work with a single set of HMMs that can be deeply optimized, instead of working with multiple sets, each of which would need to be fine tuned.

There are a number of situations in which restrictedbandwidth compensation would be advantageous with respect to other options. Portable systems such as PDAs or mobile phones are likely to receive different bandwidth input signals (eg., downloading media content from the Internet). Small footprint ASR systems are desirable due to the memory limitation of most of these portable devices. Thus, storing multiple HMM sets would not be a valid option.

Our approach would be useful in spoken document retrieval, too. The National Gallery of the Spoken Word is a project containing historical speech records from the past 100 years, sampled at different rates [5]. Broadcast news recordings may as well contain audio with different bandwidths, for example when the news anchor talks to a field correspondent. Such rapidly changing environments may benefit from our approach if either a means for automatic filtered band's detection is implemented, or a multi-bandwidth correction method can be successfully applied.

Finally, on-board car ASR [6] and extraction of the gist of activity in air traffic control [7] (where small airplanes normally transmit in limited bandwidths), are other situations where a compensation scheme for the input feature vector is desirable.

\section{Previous Work}

The algorithm presented in this paper is an evolution of our previous work on polynomial corrector functions as a means of compensation for MFCC parameters [8]. Stereo data (fulland restricted-bandwidth versions of train files) are used to obtain polynomial functions that map the restricted-bandwidth realizations to those in the full-bandwidth space; we call them 
Polynomial Corrector Functions (PCFs). It was argued in our previous work that different PCFs should be created based on the energy distribution contained in the frequency regions of the available bandwidth. We formerly created a different set of PCFs (one PCF for each cepstral coefficient) for each phoneme used in our ASR system (phoneme-based PCFs) and showed that if transcriptions were available, almost all the full-bandwidth information needed for ASR could be recovered (recognition rates were very close to the fullbandwidth test situation, even for filters as restrictive as lowpass $2 \mathrm{kHz}$ ). However, phone-based PCFs are difficult to use in real situations because no a-priori information is available regarding which phoneme-based PCF should be used for compensation of each frame. In this paper, the concept of phoneme-based PCF is substituted by an automatic statistical classification of the input data; instead of phoneme-based corrections we create class-based PCFs. Each class is defined by a GMM generated automatically using the training data. With this modification, no a-priori information is needed in the correction procedure. In addition, the scheme is faster and completely modular. Cheng et al. [9] already considered statistical classification of filtered data for the task of signal recovery for speech quality enhancement.

The statistical class-based correction algorithm presented is very similar to a technique developed for noise robustness known as SPLICE [10]. Its mathematical derivation assumes that the noise is additive and independent with respect to the speech process. Considering a limited-bandwidth signal, the mathematical derivation is different because the additive condition is substituted by a convolution. However, the solution used is the same in both situations. As it was indicated by Huang et al. [11] it seems that a SPLICE-like approach is a general solution for mismatch between train and test data, and may be applied to other situations of additive and non-additive distortion mismatch.

\section{Modeling the effect of filtering}

In this section we show the effect of bandwidth filtering over a front end of MFCCs. The unfiltered signal's MFCC representation is:

$x_{i}=\sqrt{\frac{2}{26}} \sum_{j=1}^{26} \log \left(\right.$ fbank $\left._{j}\right) \cos \left(\frac{\pi i}{26}(j-0.5)\right)$

where $i$ is the order of the MFCC. The filtered signal's MFFCs are:

$y_{i}=\sqrt{\frac{2}{26}} \sum_{j=1}^{26} \log \left(a_{j} \cdot\right.$ fbank $\left._{j}+e_{j}\right) \cos \left(\frac{\pi i}{26}(j-0.5)\right)(2)$

where $a_{j}$ is the filtering term, and $e_{j}$ is an error term introduced by the effect of non ideal filtering and finiteprecision effects.

Eq.(2), is a particular case of channel distortion with additive noise. Huang's [11] treatment of the problem shows that the vector of full-bandwidth MFCCs is related to the filtered version by:

$\vec{x}=\vec{y}-\vec{h}-\mathbf{C} \ln \left(1-e^{\mathbf{C}^{-1}(\vec{n}-\vec{y})}\right)$

where $\vec{x}, \vec{y}$ and $\vec{n}$ are the clean speech, filtered speech and noise MFCC vectors, respectively, and $\mathbf{C}$ is the DCT matrix.
This solution is a non-linear function that is difficult to consider. In addition, $\vec{h}$ is the vector of MFCC transformation of the filtering coefficients $a_{j}$. When these tend to zero (complete filtering of a frequency band), the logarithm value tends to $-\infty$, which is another source of error. Also, $\vec{n}$ is the cepstrum vector of the error terms $e_{j}$, which is again unpredictable. The approach suggested by Huang is to model the probability distribution of the noisy MFCC as a combination of GMMs that we call partitioning classes of the filtered space:

$$
\begin{aligned}
p(\vec{y}) & =\sum_{k} p(\vec{y} \mid k) \cdot P(k)= \\
& =\sum_{k} N\left(\vec{y}, \vec{\mu}_{k}, \boldsymbol{\Sigma}_{k}\right) \cdot P(k)
\end{aligned}
$$

where $P(k)$ is the a-priori probability of GMM class $k$.

Within each class, a certain stability is expected, so $\vec{x}$ and $\vec{y}$ can be assumed jointly Gaussian. Next, the expected value of $\vec{x}$ given $\vec{y}$ and class $k$ is:

$$
\begin{gathered}
\vec{x}(\vec{y}, k)=E\{\vec{x} \mid \vec{y}, k\}=\mu_{\vec{x}}^{k}+\Sigma_{\vec{x} \vec{y}}^{k}\left(\Sigma_{\vec{y}}^{k}\right)^{-1}\left(\vec{y}-\mu_{\vec{y}}^{k}\right)= \\
=\boldsymbol{B}_{k} \vec{y}+b_{0}^{k}
\end{gathered}
$$

The estimation of matrix $B_{k}$ and the constant $b_{0}^{k}$ is complex and given the error terms cited before, a precise computation does not necessarily mean better performance. Assuming a small degradation, the matrix is in many cases substituted by the identity matrix. This is equivalent to modeling the difference between the full- and limited bandwidth spaces as a set of offsets. In this work, we compare results using this simplification and those assuming a diagonal matrix. In the latter case, stereo data (clean and filtered realizations of the same speech frames) is used to calculate the diagonal matrix coefficients by linear regression. Thus, the full-bandwidth MFCC coefficients $x_{i}$ estimated from the limited-bandwidth MFCCs $y_{i}$ and class $k$ are:

$$
x_{i}\left(y_{i}, k\right)=b_{i}^{k} y_{i}+a_{i}^{k}
$$

The clean vector is then obtained using a PCF of first order (linear). We have also evaluated the possibility of extending the PCFs to higher order polynomial fits, as we did in our previous work for phoneme-dependent PCFs.

\section{Class-based partition of the filtered space}

As it was stated in Section 2, the filtered space is partitioned by a set of GMM classes. For each, a different set of PCFs (one for each cepstral coefficient) is trained. Dividing the filtered space using a number of GMMs shows several advantages over the option of modeling it in terms of phonemes as we did in our previous work [8]. GMM definition and training is done automatically based on training data. This is more natural to the system, as it does not rely on phonemes that make sense from a linguistic point of view, but 
whose significance is only relative in terms of purely acoustical characteristics. Also, filtered space automatic class partitioning does not require any knowledge of the speech or the filter characteristics and is directly applicable to unlabelled filtered test data.

\subsection{Class creation and training}

Initially, all the training frames of the filtered space are pooled together and a single mixture Gaussian model is created. Two single mixture Gaussian classes are generated, keeping the original covariance matrix and perturbing the means by plus/minus 0.2 times the standard deviation. Means and covariances are updated using the EM algorithm and this process is repeated with the introduction of a new singleGaussian class until the desired final number of classes is attained. At each step, we split the class with the smallest normalized total likelihood. These models are the seeds for the restricted-bandwidth space partitioning classes.

Once classes have been created, they are optimized by successive iterations of retraining and incrementing the number of Gaussian mixtures using again the EM algorithm.

\section{Corrector functions}

As explained in Section 3, stereo data (clean and filtered versions of the same frames) are used to obtain the unknown coefficients in Eq.(5) that map the full-bandwidth and filtered spaces.

Each frame in the training database is assigned to one of the classes dividing the filtered space:

$$
\begin{aligned}
\hat{k} & =\underset{k}{\arg \max }(p(k \mid \vec{y}))= \\
& =\underset{k}{\arg \max }\left(\frac{p(\vec{y} \mid k) \cdot P(k)}{\sum_{s} p(\vec{y} \mid s) \cdot P(s)}\right)= \\
& =\underset{k}{\arg \max }(p(\vec{y} \mid k) \cdot P(k))
\end{aligned}
$$

where $P(k)$ is the a-priori probability of each GMM class which we keep equal for all GMMs. The likelihood for each GMM class is:

$$
p(\vec{y} \mid k)=\sum_{i}^{M-1} N\left(\vec{y}, \vec{\mu}_{i}^{k}, \Sigma_{i}^{k}\right) \cdot P_{i}^{k}
$$

where $N\left(\vec{y}, \vec{\mu}_{i}^{k}, \Sigma_{i}^{k}\right)$ is the Gaussian probability and $P_{i}^{k}$ the a-priori probability of a given Gaussian mixture.

For each class and each cepstrum coefficient, the two realizations of each frame are mapped. In Section 3, two different simplifications of Eq.(5) were discussed: corrector offsets and PCFs. Corrector offsets are calculated as the mean difference between the two realizations of each frame:

$\vec{r}_{i}^{k}=\frac{1}{N_{k}} \sum_{j}^{N_{k}} \vec{x}_{j}^{k}-\vec{y}_{j}^{k}$

For PCFs, coefficients that determine a least squares regression are used.
Table 1: ASR results.

\begin{tabular}{|c|c|c|c|c|c|}
\hline & $\begin{array}{c}\text { Train } \\
\text { Bwidth }\end{array}$ & $\begin{array}{c}\text { Test } \\
\text { Bwidth }\end{array}$ & Correction & $\begin{array}{c}\text { Percent } \\
\text { Corr. } \\
\text { (\%) }\end{array}$ & $\begin{array}{c}\text { Word } \\
\text { Acc. } \\
\text { (\%) }\end{array}$ \\
\hline A & $8 \mathrm{kHz}$ & $8 \mathrm{kHz}$ & No & 78.79 & 76.20 \\
\hline $\mathrm{B}$ & $8 \mathrm{kHz}$ & $4 \mathrm{kHz}$ & No & 30.22 & 20.54 \\
\hline $\mathrm{C}$ & $4 \mathrm{kHz}$ & $4 \mathrm{kHz}$ & No & 71.55 & 67.44 \\
\hline $\mathrm{D}$ & $8 \mathrm{kHz}$ & $4 \mathrm{kHz}$ & CMN & 63.67 & 58.78 \\
\hline $\mathrm{E}$ & $8 \mathrm{kHz}$ & $4 \mathrm{kHz}$ & $\begin{array}{c}\text { PCFs. } \\
\text { (Phoneme Based) }\end{array}$ & 71.36 & 67.61 \\
\hline $\mathrm{F}$ & $8 \mathrm{kHz}$ & $4 \mathrm{kHz}$ & $\begin{array}{c}\text { PCFs. } \\
\text { (Class Based) }\end{array}$ & 74.78 & 71.51 \\
\hline
\end{tabular}

\section{Evaluation}

Evaluation is based on recognition rates using an HMM-based ASR engine consisting of 51 models ( 3 emitting states with 15 Gaussian mixtures per state) including short-pause and starting and ending silences. The front-end uses pre-emphasis filtering $(\alpha=0.97)$ and $25 \mathrm{~ms}$ Hamming windows with a $10 \mathrm{~ms}$ window shift. Thirteen MFCC coefficients along with $\mathrm{C} 0$ and their respective first and second order derivatives are computed from a filter-bank of 26 triangular filters uniformly distributed in the Mel-Frequency scale along the region 0-8 kHz. The entire training partition of TIMIT is used for training of the HMMs and creation and training of the GMM classes that partition the filtered space and calculation of the corrector offsets and PCFs' coefficients. Speech recognition accuracy rates are given for the entire test set in TIMIT. Experiments are carried out using HTK tools [12].

Table 1 shows results for different approaches to the problem of full-bandwidth-trained models and restrictedbandwidth (low-pass $4 \mathrm{kHz}$ ) input test data. The grammar factor was fixed for all experiments to the value that maximizes the full-bandwidth system's accuracy (fullbandwidth train and test data). This accounts for the small recognition rate differences with equivalent experiments in our previous work [8].

The baseline system's rates (A) are given as an indicator of the overall performance of the recognizer architecture. However, base system's rates are arguably unattainable for the task of unmatched train and test data given the loss of information contained in the filtered bands. A comparison between baseline rates and those of the re-trained models with matched conditions show a relative difference of about $9 \%$ for the case of low-pass filtered $4 \mathrm{kHz}$ test data.

The class-based PCFs method's percent correct and word accuracy rates $(\mathrm{F})$ beat those of the matched train-test data situation (C) by a relative 4\%. The fact that the PCFs method outperforms the matched case is somehow surprising. The reasons are still unclear and although we propose an explanation later, more experiments need to be carried out to confirm our hypothesis. Nevertheless, Deng et al. [13] reported the same conclusion for noisy data experiments: the matched HMMs do not necessarily set the upper limit on performance.

Table 2 provides an analysis on the results of Table 1 and suggests reasons for better performance of our approach. The impression is that the difference between the cases $\mathrm{C}$ and $\mathrm{F}$ (matched against 'unmatched + PCFs') is worse HMM 
Table 2: Analysis of results. Letters A, B, C and F refer to the left-most column of Table 1.

\begin{tabular}{|c|l|}
\hline \multicolumn{1}{|c|}{ Situation } & \multicolumn{1}{c|}{ Reasons } \\
\hline Degrad. A to B & $\begin{array}{l}\text { 1. Train-test mismatch. } \\
\text { 2. Loss of information in test data. }\end{array}$ \\
\hline Degrad. A to C & $\begin{array}{l}\text { 1. Loss of information in train } \\
\text { data } \rightarrow \text { Worse HMM models. } \\
\text { 2. Loss of information in test data. }\end{array}$ \\
\hline Degrad. A to F & $\begin{array}{l}\text { 1. Train-test mismatch (but reduced). } \\
\text { 2. No loss of information in train } \\
\text { data } \rightarrow \text { Good HMM models. } \\
\text { 3. Loss of information in test data. }\end{array}$ \\
\hline
\end{tabular}

training versus reduced mismatch; loss of information of training data is a common flaw for any approach. Recognition rates in Table 1 seem to indicate that the mismatch is successfully mitigated so that the PCFs perform better than the matched HMM models. However, similar experiments on other databases are planned to further extend our conclusions, and to investigate the reasons.

Table 3 shows a comparison between the two correcting solutions: simple offsets and PCFs of different orders. We conclude that the polynomial fit correction performance shows almost no dependence with the fit order; contrary to our first impressions on experiments on phoneme-based corrector functions, class-based corrector functions show no remarkable advantage on using second or higher order polynomial fits. When simple offsets are considered (corresponding to an order 0 polynomial fit) Eq. (9) is used instead of a linear regression fit. In this case, again, no substantial difference is observed although a statistical experiment (ANOVA) suggested that a first order fit would provide benefits over simple offsets.

\section{Conclusions and future work}

The PCFs' schema has proved successful in estimating the differences between the restricted-bandwidth and fullbandwidth MFCC spaces. Thus, we show that for this particular case of channel distortion, an optimized HMM set trained with full-bandwidth data and a set of PCFs may be more effective on limited-bandwidth test data than a new HMM set trained with matched data. This situation may seem odd as it is normally assumed that the best training method is the one matching the test conditions. Our approach seems to benefit from better HMM modeling thanks to full-bandwidth training data (full-bandwidth HMM training probably allows for a more effective alignment of phonemes than the restricted-bandwidth situation), while the problem of unmatched train-test data seems to be minimized by the compensator functions. Data-driven creation of GMM classes for partitioning the filtered space is effective as it does not rely on a-priori knowledge of the acoustic space, as would be the case with phoneme-based partitioning of the space.

The algorithm proposed in this paper is a general feature enhancement based solely on training data. This is important in the sense that the problem may be generalized to any kind of band-filtering, and also to different channel distortion problems. However, as the case of low pass filtering $4 \mathrm{kHz}$ is of special practical significance due to the specifications of telephone voice, our future work will emphasize more
Table 3: Offset and different orders of polynomial fit's results. HMMs trained with full-bandwidth data. Test data: low pass filtered $4 \mathrm{kHz}$.

\begin{tabular}{|c|c|c|}
\hline Order & $\begin{array}{c}\text { Percent } \\
\text { Correct (\%) }\end{array}$ & $\begin{array}{c}\text { Word } \\
\text { Accuracy (\%) }\end{array}$ \\
\hline Offset & 74.84 & 71.48 \\
\hline Linear Fit & 74.78 & 71.51 \\
\hline Second Order Fit & 74.70 & 71.65 \\
\hline Third Order Fit & 75.08 & 71.99 \\
\hline Fourth Order Fit & 74.79 & 71.54 \\
\hline Fifth Order Fit & 74.68 & 71.60 \\
\hline
\end{tabular}

specific corrections accounting for the known difficulties of discerning between phonemes that have their differentiating information in the higher frequencies (fricatives or plosives). Experiments were carried out using synthetic data (filtering clean data in the laboratory). In the future we expect to extend the success of this technique to real data and in particular to the task of spoken document retrieval.

\section{References}

[1] H. Yasukawa, "Restoration of wide band signal from telephone speech using linear prediction error processing”, Proc. ICSLP'96, 901-904.

[2] L. Rabiner and B. Juang, Fundamentals of Speech Recognition, Prentice Hall, New Jersey, 1993.

[3] S. Chennoukh, A. Gerrits, G. Miet and R. Sluijter, "Speech enhancement via frequency bandwidth extension using line spectral frequencies", Proc. ICASSP'01, (1): $665-668$.

[4] J.G. Fiscus, "A post-processing system to yield reduced word error rates: Recognizer Output Voting Error Reduction (ROVER)", Proc. ASRU'97. 347 - 354.

[5] http://www.ngsw.org/

[6] H. Abut, J.H.L Hansen and K. Takeda (eds.), DSP for InVehicle and Mobile Systems, Kluwer/Springer-Verlag. 2005.

[7] L. Denenberg, H. Gish, M. Meteer, T. Miller, J.R. Rohlicek, W. Sadkin and M. Siu, "Gisting conversational speech in real time”, Proc. ICASSP'93, (2): 131 - 134.

[8] N. Morales, J.H.L. Hansen and D.T. Toledano, "MFCC Compensation for Improved Recognition of Filtered and Band-Limited Speech", Proc. ICASSP'05, (1): 521-524.

[9] Y.M. Cheng, D. O'Shaughnessy, P. Mermelstein, "Statistical recovery of wideband speech from narrowband speech", Speech and Audio Processing, IEEE Trans., (2): 4.544 - 548, Oct. 1994.

[10] J. Droppo, A. Acero and L. Deng. "Evaluation of the SPLICE Algorithm on the Aurora2 Database", Proc. Eurospeech'01. 217-220.

[11] X. Huang, A. Acero and H. W. Hon, Spoken Language Processing: A Guide to Theory, Algorithm and System Development, Prentice Hall PTR, 2001.

[12] S. Young, G. Evermann, T. Hain, D. Kershaw, G. Moore, J. Odell, D. Ollason, D. Povey, W. Valtchev and P Woodland, The HTK Book (for HTK version 3.2.1), December 2002.

[13] L. Deng, A. Acero, M. Plumpe and X. Huang, "LargeVocabulary Speech Recognition Under Adverse Acoustic Environments", Proc. ICSLP'00, (3): 806-809. 